\title{
Efeitos da frequência de fornecimento do volumoso e concentrado no comportamento ingestivo de vacas e novilhas em confinamento
}

\section{Raul Dirceu Pazdiora1 ${ }^{1}$, Ivan Luiz Brondani ${ }^{2}$, Magali Floriano da Silveira ${ }^{3}$, Miguelangelo Ziegler Arboitte ${ }^{4}$, Jonatas Cattelam ${ }^{5}$, Perla Cordeiro de Paula ${ }^{6}$}

\author{
1 Doutorando do PPGZ - FCAVIUNESP \\ 2 Departamento de Zootecnia - UFSM. \\ 3 Doutoranda do PPGZ - UFSM \\ 4 Instituto Federal Catarinense (IFC - Sombrio). \\ 5 Acadêmico de Medicina Veterinária - UFSM. \\ ${ }^{6}$ Acadêmica de Zootecnia - UFSM.
}

RESUMO - Este estudo foi conduzido com o objetivo de avaliar a influência da frequência no fornecimento de volumoso e concentrado e/ou da categoria animal sobre o comportamento ingestivo de bovinos. Foram utilizadas 16 novilhas e 16 vacas com idade média inicial de 20 e 66 meses e peso médio inicial de 338 e 432 kg, respectivamente. Os tratamentos foram representados pelas frequências de fornecimento do volumoso e do concentrado: 2 V/C - volumoso e concentrado duas vezes ao dia; $1 \mathrm{~V} / \mathrm{C}$ - volumoso e concentrado uma vez ao dia; $1 \mathrm{~V} / 2 \mathrm{C}$ - volumoso uma vez ao dia e concentrado duas vezes ao dia; 1 V/3 C - volumoso uma vez ao dia e concentrado três vezes ao dia, ofertadas às categorias vacas e novilhas. A dieta foi composta de $60 \%$ de silagem de milho e $40 \%$ de concentrado com base na matéria seca (MS). O delineamento experimental foi inteiramente casualizado em arranjo fatorial $4 \times 2$ (frequências $\times$ categorias). Os dados foram submetidos à análise de variância e as médias, comparadas pelo teste t. A interação frequência de fornecimento e categoria animal foi significativa para as características tempo de ruminação e ócio, eficiência de ruminação da matéria seca e da fibra detergente neutro, número de bolos diário e tempo diário de mastigação. Em relação às vacas, as novilhas apresentaram maiores tempos de ócio e mastigação quando receberam a alimentação uma vez ao dia. A frequência de fornecimento da dieta não influenciou o tempo de consumo. As vacas apresentaram maior tempo consumindo alimento e taxa de ingestão em comparação às novilhas (4,38 vs 4,09 horas e 2,91 vs 2,35 kg de MS/hora de consumo, respectivamente). O aumento na frequência da alimentação estimula os animais a maior ingestão de alimento no momento do fornecimento.

Palavras-chave: categoria animal, consumo de alimento, manejo da alimentação, mastigações, ócio, ruminação

\section{Effects of supply frequency of roughage and concentrate on ingestive behavior of feedlot cows and heifers}

ABSTRACT - The present study was conducted with the objective of evaluating the influence of frequency of roughage and supplement supply and/or animal category on the ingestive behavior of cattle. Sixteen heifers and sixteen cows, with average initial age of 20 and 66 months and average initial weight of 338 and $432 \mathrm{~kg}$, respectively, were used. Treatments were represented by the frequencies of roughage and concentrate supply: 2 R/C - roughage and concentrate two times per day; $1 \mathrm{R} / \mathrm{C}$ - roughage and concentrate one time per day; $1 \mathrm{R} / 2 \mathrm{C}$ - roughage one time per day and concentrate two times per day; 1 R/3 C - roughage one time per day and concentrate three times per day, offered to cows and heifers. Diet was composed of $60 \%$ maize silage and $40 \%$ concentrate, on a dry matter basis (DM). The complete randomized experimental design was used, with a $4 \times 2$ factorial arrangement (frequencies $\times$ category). The data were submitted to variance analysis and averages were compared by "t" test. The interaction between supply frequencies and animal category was significant for rumination and idle times, efficiencies of dry matter rumination and of neutral detergent fiber rumination, number of ruminated bolus and daily time of chew. Heifers showed longer idle periods and daily time of chew when compared with cows that received food once in a day. Duration of feed intake did not vary with frequencies. Cows showed longer period of feed intake and ingestive rate in relation to heifers (4.38 vs. 4.09 hours and $2.91 \mathrm{vs} .2 .35 \mathrm{~kg}$ of DM/hour of intake, respectively). The increase in food frequency stimulated animals to intensify feed intake at the moment of supply.

Key Words: animal category, chews, food handling, food intake, idleness, rumination 


\section{Introdução}

As atividades diárias dos animais ruminantes estabulados são caracterizadas por três comportamentos básicos: alimentação, ruminação e ócio, sendo os períodos gastos com a ingestão de alimentos intercalados com períodos de ruminação ou de ócio. O tempo gasto em ruminação é normalmente mais prolongado à noite, mas os períodos de ruminação são ritmados também pelo fornecimento de alimento. No entanto, existem diferenças individuais quanto à duração e à repartição das atividades de ingestão e ruminação, que parecem estar relacionadas ao apetite, às diferenças anatômicas e ao suprimento das exigências energéticas ou repleção ruminal, estas influenciadas pela relação volumoso:concentrado, pelas condições climáticas (Fischer et al., 2002), pelo manejo e atividade dos animais em grupo (Fischer et al., 1997).

A duração do tempo de alimentação no cocho, associada aos horários que preferencialmente os animais exercem a alimentação, é importante para estabelecer estratégias de manejo adequadas para cada situação. Segundo Mendonça et al. (2004), o estudo do comportamento ingestivo possibilita o ajuste do manejo alimentar para obtenção do melhor desempenho produtivo.

Variações no consumo de alimento podem ser evidenciadas em avaliação do comportamento alimentar. Os objetivos quando se estuda o comportamento ingestivo dos animais são avaliar os efeitos do arraçoamento ou a quantidade e qualidade nutritiva do alimento; estabelecer a relação entre comportamento ingestivo e consumo voluntário; e averiguar o uso potencial do conhecimento sobre o comportamento ingestivo para a melhoria do desempenho animal (Lima et al., 2003). No entanto, o volume de informações na literatura ainda não oferece dados conclusivos a respeito da resposta animal em distribuição das atividades ingestivas quando esses recebem alimentação em diferentes frequências no decorrer do dia. Neste sentido, o estudo foi conduzido com o objetivo de avaliar se a frequência do fornecimento do volumoso e concentrado e/ ou a categoria animal afetam o comportamento ingestivo de bovinos confinados.

\section{Material e Métodos}

O experimento foi conduzido no Laboratório de Bovinocultura de Corte do Departamento de Zootecnia da Universidade Federal de Santa Maria (UFSM), situado na região denominada Depressão Central do Rio Grande do Sul, no período de maio a agosto de 2006.
O confinamento teve duração de 79 dias, sendo 16 dias de adaptação dos animais às instalações, manejo e alimentação e 63 dias de avaliações. Foram utilizadas 16 novilhas com idade média de 20 meses e peso médio de $338 \mathrm{~kg}$ com desvio padrão de 29,9 e 16 vacas com idade média de 66 meses e $432 \mathrm{~kg}$ de peso com desvio padrão de 26,8 ao início do período de avaliação, sendo os animais cruzas Charolês - Nelore.

Os animais foram confinados em baias de $20 \mathrm{~m}^{2}$ parcialmente cobertos, com piso de alvenaria, com bebedouro regulado por torneira-bóia e disponibilidade de $2 \mathrm{~m}$ linear de cocho por animal. Todos os boxes receberam dois bovinos da mesma categoria.

Foram adotados quatro manejos alimentares conforme as frequências do fornecimento de volumoso e concentrado, que correspondem aos tratamentos: $2 \mathrm{~V} / \mathrm{C}$ - volumoso e concentrado duas vezes ao dia fornecidos em parte iguais, às 8 e às $18 \mathrm{~h} ; 1 \mathrm{~V} / \mathrm{C}$ - volumoso e concentrado uma vez ao dia, às 8 horas; $1 \mathrm{~V} / 2 \mathrm{C}$ - volumoso uma vez ao dia, às $8 \mathrm{~h}$, e o concentrado fornecido duas vezes ao dia, às 8 e às $18 \mathrm{~h}$ ); $1 \mathrm{~V} / 3 \mathrm{C}$ - volumoso uma vez ao dia, às $8 \mathrm{~h}$, e o concentrado fornecido três vezes ao dia, às 8,13 e 18 h, ofertados as categorias vaca e novilha.

A dieta fornecida aos animais foi composta de $60 \%$ de silagem de milho e $40 \%$ de concentrado com base na matéria seca (MS), misturados no cocho, com o objetivo inicial de atender à exigência de proteína bruta (PB) para ganho de peso médio diário (GMD) de 1,200 kg (NRC, 1996), estimando consumo de 2,5 kg de MS/100 kg de peso corporal (PC) (Tabela 1).

Tabela 1 - Composição das dietas utilizadas no período experimental

\begin{tabular}{lcc}
\hline Ingrediente (\%) & \multicolumn{2}{c}{ Categoria } \\
\cline { 2 - 3 } & Novilhas & Vacas \\
\hline Silagem de milho & 60,0 & 60,0 \\
Grão de milho & 10,0 & 6,0 \\
Farelo de trigo & 16,2 & 22,44 \\
Farelo de glúten de milho & 12,0 & 10,0 \\
Ureia & 0,48 & 0,32 \\
Calcário calcítico & 0,88 & 0,82 \\
Cloreto de sódio & 0,40 & 0,40 \\
Rumensin ${ }^{\circledR}$ & 0,016 & 0,012 \\
Sulfato de amônia & 0,03 & 0,02 \\
Composição nutricional (\%) & & \\
Matéria seca & 54,91 & 54,97 \\
Matéria orgânica & 90,09 & 90,07 \\
Proteína bruta & 12,89 & 12,10 \\
Extrato etéreo & 3,19 & 3,14 \\
Fibra em detergente neutro & 35,87 & 32,90 \\
Fibra em detergente ácido & 18,49 & 19,45 \\
Carboidratos não-fibrosos & 38,14 & 41,93 \\
Nutrientes digestíveis totais & 71,53 & 71,66 \\
\hline Valores expressos & &
\end{tabular}

Valores expressos em $100 \%$ da matéria seca. 
O consumo da dieta foi registrado diariamente, sendo realizada a pesagem da quantidade de alimentos fornecidos e das sobras de alimentos do dia anterior. A oferta de alimentos foi estipulada em $10 \%$ acima do consumo voluntário, regulada de acordo com o consumo do dia anterior.

Foram coletadas, semanalmente, amostras representativas dos componentes da dieta alimentar durante o período experimental. As amostras foram pré-secas em estufa de ar forçado a $55^{\circ} \mathrm{C}$, por 72 horas para determinação da matéria parcialmente seca. Posteriormente, foram processadas em moinho tipo Willey com peneira com crivos de $1 \mathrm{~mm}$ e armazenadas para posterior análise laboratorial. Foram determinados os teores de MS e matéria orgânica (MO), $\mathrm{PB}$, extrato etéreo (EE) e cinzas (MM), segundo (AOAC, 1995).

A determinação da fibra em detergente neutro (FDN) e fibra em detergente ácido (FDA) foi feita em saquinhos de poliéster (Komarek, 1993). Os teores de carboidratos nãofibrosos (CNF) foram estimados segundo Mertens (1997), sendo CNF: 100 - (FDN\% + EE\% + PB\% + MM\%). O teor de nutrientes digestíveis totais (NDT) foi calculado segundo Weiss et al. (1992).

Foram avaliadas as distribuições das atividades ingestivas dos animais ao longo do dia. Para o registro do tempo destinado ao consumo de alimento e água, ruminação e ócio adotou-se a observação visual dos animais a cada cinco minutos, durante 48 horas consecutivas, três vezes ao longo do período experimental. Durante as observações noturnas, o ambiente foi mantido com iluminação artificial.

As médias do número de mastigações por bolo ruminal (NMB) e do tempo de mastigações por bolo ruminal (TMB) foram obtidas pela cronometragem de 20 observações por repetição em cada dia de avaliação.

Os resultados referentes aos fatores do comportamento ingestivo foram obtidos pelas relações adaptadas de Bürger et al (2000), nas quais: TI = taxa de ingestão (kg MS/h); CMS = consumo de matéria seca (kg MS/dia); TCA = tempo consumindo alimento (horas/dia); ER = eficiência deruminação (kg MS/hora); TR = tempo de ruminação (horas/dia); TMD = tempo de mastigação diária (horas/dia); NBD = número de bolos mastigados por dia ( $\mathrm{n} / \mathrm{dia}) ; \mathrm{TMB}=$ tempo destinados a mastigação por bolo ruminal (seg/bolo); NMD = número de mastigadas mericíclicas diárias (nºdia) e TO = tempo ao ócio (horas/dia), foram obtidos pelas seguintes equações, em que: $\mathrm{TI}=\mathrm{CMS} / \mathrm{TCA}$; $\mathrm{ER}=\mathrm{CMS} / \mathrm{TR}$; $\mathrm{NBD}=\mathrm{TR} / \mathrm{TMB} ; \mathrm{NMD}=\mathrm{NMB} * \mathrm{NBD}$ e TMD $=\mathrm{TR}+\mathrm{TCA}$.

O delineamento experimental utilizado foi o inteiramente casualizado em arranjo fatorial $4 \times 2$ (4 frequências de fornecimento e 2 categorias de bovinos). Os dados foram submetidos à análise de variância e as médias comparadas pelo teste t de Student (PDIFF) com auxílio do programa estatístico SAS (2001), a 5\% de significância, utilizando-se o seguinte modelo matemático:

$Y_{i j k}=\mu+\alpha_{i}+\beta_{j}+(\alpha * \beta)_{i j}+\varepsilon_{i j k}$,

em que $\mathrm{Y}_{\mathrm{ijk}}=$ variáveis dependentes; $\mu$ = média geral de todas as observações; $\alpha_{i}=$ efeito da i-ésima frequência de fornecimento de ordem 1 para 2 V/C; 2 para $1 \mathrm{~V} / \mathrm{C}$; 3 para $1 \mathrm{~V} / 2 \mathrm{C}$; 4 para $1 \mathrm{~V} / 3 \mathrm{C} ; \beta_{\mathrm{j}}=$ efeito da j-ésima categoria de ordem 1 para vacas; 2 para novilhas; $(\alpha * \beta)_{\mathrm{ij}}=$ interação entre a i-ésima frequência de fornecimento e a j-ésima categoria; $\varepsilon_{\mathrm{ijk}}=$ erro aleatório residual, $\operatorname{NID}\left(0, \sigma^{2}\right)$.

\section{Resultados e Discussão}

Não houve interação para o CMS em peso absoluto e em relação ao peso corporal, consumo de fibra detergente neutro (CFDN) e GMD (Tabela 2).

Os animais que receberam o volumoso uma vez ao dia e o concentrado fracionado em 2 ou 3 vezes apresentaram maior consumo de MS em peso absoluto em relação aos demais. Quando analisado em relação ao peso corporal (não houve diferença), o maior consumo de MS não promoveu diferenças no ganho médio diário. O consumo de FDN apresentou comportamento semelhante ao consumo de MS, em que os animais que receberam o volumoso uma vez ao dia e o concentrado fracionado 2 vezes consumiram mais FDN em relação a um fornecimento do volumoso e

Tabela 2 - Consumo diário de matéria seca por animal (CMS) e em porcentagem de peso corporal (CMSPC), consumo de fibra detergente neutro (CFDN) e ganho médio em peso (GMD) conforme a frequência do fornecimento e a categoria animal

\begin{tabular}{|c|c|c|c|c|c|c|}
\hline \multirow[t]{2}{*}{ Variável } & \multicolumn{4}{|c|}{ Frequência diária de fornecimento da dieta dia } & \multicolumn{2}{|c|}{ Categoria } \\
\hline & $2 \mathrm{~V} / \mathrm{C}$ & $1 \mathrm{~V} / \mathrm{C}$ & $1 \mathrm{~V} / 2 \mathrm{C}$ & $1 \mathrm{~V} / 3 \mathrm{C}$ & Novilhas & Vacas \\
\hline CMS, kg/dia & $10,6 b$ & $10,7 \mathrm{~b}$ & $11,7 \mathrm{a}$ & $11,4 \mathrm{a}$ & $9,57 b$ & $12,65 \mathrm{a}$ \\
\hline CFDN, kg/dia & $4,77 b$ & $4,80 \mathrm{~b}$ & $5,29 a$ & 4,99ab & $4,10 \mathrm{~b}$ & $5,82 \mathrm{a}$ \\
\hline GMD, kg/dia & 1,265 & 1,360 & 1,486 & 1,307 & 1,330 & 1,378 \\
\hline
\end{tabular}

a, b Médias seguidas de letras diferentes para o mesmo parâmetro, diferem entre si $(\mathrm{P}<0,05)$;

$\mathrm{V}=$ volumoso.

$\mathrm{C}=$ concentrado 
concentrado ou o fracionamento destes em 2 vezes ao dia, não diferindo do fornecimento de uma vez do volumoso e o concentrado fracionado 3 vezes. Quanto à categoria, as vacas apresentaram maiores consumos de MS e FDN, no entanto, o consumo expresso em relação ao peso corporal e o GMD não diferiu entre as categorias.

Houve interação significativa entre frequência de fornecimento e categoria animal para os tempos de ócio e de ruminação e as eficiências da ruminação da MS e da FDN (Tabela 3).

As novilhas apresentaram maior $(\mathrm{P}<0,05)$ tempo de ócio em relação às vacas, quando receberam a alimentação uma vez ao dia. Já para a categoria das vacas, aquelas que receberam o volumoso uma vez e o concentrado fracionado em duas ou três vezes ao longo do dia permaneceram mais tempo em ócio em relação às que receberam a alimentação somente uma vez ao dia ou o volumoso e o concentrado fracionado em duas vezes, corroborando com Deswysen et al. (1993) e Fischer et al. (1997), em que relatam que os períodos de ruminação e ócio entre as refeições, sua duração e seu padrão de distribuição são influenciados pelas atividades de ingestão. O ócio é considerado o tempo que o animal fica sem atividade física, podendo estar deitado ou em pé.

O tempo de ruminação foi menor para as vacas que receberam um fornecimento do volumoso e o concentrado fracionado duas ou três vezes ao longo do dia. Esse tempo dedicado à ruminação está diretamente relacionado à qualidade e à quantidade de alimento consumido (Mendes et al., 2010). Diversos autores citados por Mertens (1997) demonstraram que a atividade de ruminação é uma característica que reflete as propriedades físicas e químicas dos alimentos, como a concentração de FDN, o tamanho de partículas e a umidade. Em condições normais a campo, vários autores encontraram menor tempo dedicado a ruminação do que o tempo de pastejo (Soares et al., 2006; Zanine et al., 2007; Monzano et al., 2007). No entanto, no presente trabalho que foi realizado com animais confinados, observa-se que o tempo de ruminação foi superior em $195,5 \%$ do tempo gasto de ingestão do alimento, valor semelhante ao encontrado por Ferreira (2006) e Missio et al. (2010), demonstrando que o tempo de ruminação em confinamento é superior. Isto ocorre em função do tempo de pastejo no campo, pela seleção do pasto, ser maior que o de alimentação em confinamento (Souza et al., 2007). A diminuição do tempo destinado à ruminação e o aumento do tempo de descanso dos animais, conforme observado na Tabela 3 (vacas recebendo 1 V/2 C e 1 V/2 C) são importantes, pois implicam na diminuição de atividade física, que demanda energia (Missio et al., 2010), acarretando maiores quantidades de energia para ganho em substituição a energia requerida para a mantença, que segundo o NRC (2001) pode ser de 10 a $20 \%$ superior em animais em pastejo.

Em relação à eficiência de ruminação da MS, esta foi maior para as vacas em relação às novilhas para todas as frequências de fornecimento do volumoso e concentrado, em função das novilhas apresentarem menor CMS e maior tempo de ruminação. Mesma variação foi observada para a eficiência de ruminação da FDN. Segundo Van Soest (1994), o tempo despendido em ruminação, influenciado pela

Tabela 3 - Médias e erros-padrão (EP) dos tempos de ócio e ruminação e eficiência de ruminação da matéria seca e da fibra detergente neutro

\begin{tabular}{|c|c|c|c|c|c|c|}
\hline \multirow[b]{2}{*}{ Categoria } & \multicolumn{4}{|c|}{ Frequências do fornecimento da dieta } & \multirow[b]{2}{*}{ EP } & \multirow[b]{2}{*}{ Médias } \\
\hline & $2 \mathrm{~V} / \mathrm{C}$ & $1 \mathrm{~V} / \mathrm{C}$ & $1 \mathrm{~V} / 2 \mathrm{C}$ & $1 \mathrm{~V} / 3 \mathrm{C}$ & & \\
\hline \multicolumn{7}{|c|}{ Tempo de ócio, horas } \\
\hline Vaca & $10,74 b$ & $10,39 b$ & $11,88 \mathrm{a}$ & $11,85 a$ & 0,21 & $11,22 \pm 0,10$ \\
\hline Novilha & $10,65 b$ & $11,40 \mathrm{a}$ & $11,32 \mathrm{ab}$ & $11,75 a$ & 0,21 & $11,28 \pm 0,10$ \\
\hline Médias & 10,69 & 10,90 & 11,60 & 11,80 & 0,15 & \\
\hline \multicolumn{7}{|c|}{ Tempo de ruminação, horas } \\
\hline Vaca & $8,71 \mathrm{ab}$ & $8,77 \mathrm{ab}$ & 7,49 e & 7,66de & 0,18 & $8,16 \pm 0,09$ \\
\hline Novilha & $8,92 \mathrm{a}$ & $8,30 \mathrm{bc}$ & $8,11 \mathrm{~cd}$ & $8,24 \mathrm{bc}$ & 0,18 & $8,39 \pm 0,09$ \\
\hline Médias & 8,81 & 8,54 & 7,80 & 7,95 & 0,13 & \\
\hline \multicolumn{7}{|c|}{ Eficiência de ruminação da MS, kg/hora } \\
\hline Vaca & $1,38 b$ & $1,37 \mathrm{~b}$ & $1,79 \mathrm{a}$ & $1,72 \mathrm{a}$ & 0,04 & $1,56 \pm 0,02$ \\
\hline Novilha & $1,03 c$ & $1,12 \mathrm{c}$ & $1,23 \mathrm{bc}$ & $1,17 \mathrm{c}$ & 0,04 & $1,14 \pm 0,02$ \\
\hline Médias & 1,21 & 1,25 & 1,51 & 1,44 & 0,03 & \\
\hline \multicolumn{7}{|c|}{ Eficiência de ruminação da FDN, kg/hora } \\
\hline Vaca & $0,64 b$ & $0,64 b$ & $0,83 a$ & $0,76 \mathrm{a}$ & 0,02 & $0,72 \pm 0,01$ \\
\hline Novilha & $0,44 \mathrm{~d}$ & $0,48 \mathrm{~cd}$ & $0,53 c$ & $0,50 \mathrm{~cd}$ & 0,02 & $0,48 \pm 0,01$ \\
\hline Médias & 0,54 & 0,56 & 0,68 & 0,63 & 0,01 & \\
\hline
\end{tabular}

a,b,c,d,e Médias seguidas de letras diferentes para o mesmo parâmetro diferem $(\mathrm{P}<0,05)$ entre si.

$\mathrm{V}=$ volumoso.

$\mathrm{C}=$ concentrado 
natureza da dieta, é proporcional ao teor de parede celular dos volumosos (quanto maior a participação de volumosos na dieta maior o tempo despendido em ruminação). Sendo assim, a eficiência de ruminação ou mastigação pode ser reduzida em dietas com elevado tamanho de partícula e alto teor de fibra, tendo em vista a maior dificuldade para reduzir o tamanho das partículas originadas destes materiais fibrosos. Além disso, Silva et al. (2005) afirmaram que a eficiência de ruminação do alimento é afetada positivamente pela elevação da matéria seca da dieta.

Segundo Burger et al. (2000), o aumento do consumo tende a reduzir o tempo de ruminação por grama de alimento, fator provavelmente responsável pelo aumento de tamanho das partículas fecais, quando os consumos são elevados. Welch (1982) afirmou que o aumento no fornecimento de fibra indigestível não incrementa a ruminação a mais de 8 ou 9 h/dia, sendo a eficácia de ruminação importante no controle da utilização de volumosos; assim, o animal que ruminar maior quantidade de volumoso durante esse período de tempo pode consumir mais e ser teoricamente mais produtivo.

Não houve interação significativa para o número de mastigações por bolo ruminal e tempo de mastigação por bolo ruminal (Tabela 4). O número de mastigações por bolo e o tempo de mastigação por bolo não foram influenciados pela frequência de fornecimento do volumoso e do concentrado e pela categoria animal, isto se deve provavelmente pela composição dos ingredientes ser semelhante entre as dietas. A mastigação durante a ingestão e/ou a ruminação atua diretamente na redução das partículas do alimento e implica, indiretamente, nas condições ótimas para celulobiose ruminal, devido ao efeito sobre a produção de saliva.

Ferreira (2006), avaliando o comportamento de vacas e novilhos em confinamento, observou que as vacas apresentaram menor número de mastigações por bolo ruminal do que novilhos (Tabela 5). As principais consequências são a redução da atividade mastigatória, consequentemente, menor secreção de saliva, redução do $\mathrm{pH}$ ruminal e a diminuição da relação acetato:propionato (Van Soest, 1994).

O número de bolos mastigados por dia foi maior para as novilhas alimentadas uma vez com o volumoso e duas vezes com concentrado em relação às vacas (563 vs 436 bolos/dia, respectivamente). Missio et al. (2010) encontraram valores médios de 614,8; 649,3; 565,3 e 433,8 bolos ao dia, correspondentes aos níveis de 22; 40; 59 e 79\% de concentrado na dieta.

Tabela 4 - Médias e erros-padrão (EP) do número de mastigações por bolo e tempo de mastigações por bolo

\begin{tabular}{|c|c|c|c|c|c|c|}
\hline \multirow[t]{2}{*}{ Variável } & \multicolumn{4}{|c|}{ Frequência de fornecimento da dieta } & \multirow[b]{2}{*}{ EP } & \multirow[b]{2}{*}{ Médias } \\
\hline & $2 \mathrm{~V} / \mathrm{C}$ & $1 \mathrm{~V} / \mathrm{C}$ & $1 \mathrm{~V} / 2 \mathrm{C}$ & $1 \mathrm{~V} / 3 \mathrm{C}$ & & \\
\hline Número de mastigações/bolo & 62 & 55 & 57 & 56 & 2,13 & 57,5 \\
\hline \multirow[t]{3}{*}{ Tempo de mastigação/bolo, s } & 61 & 58 & 57 & 56 & 1,88 & 58,0 \\
\hline & \multicolumn{4}{|c|}{ Categorias } & & \\
\hline & \multicolumn{2}{|c|}{ Novilha } & \multicolumn{2}{|c|}{ Vaca } & & \\
\hline Número de mastigações/bolo & \multicolumn{2}{|c|}{58} & \multicolumn{2}{|c|}{56} & 1,51 & 57,0 \\
\hline Tempo de mastigação/bolo, s & \multicolumn{2}{|c|}{56} & \multicolumn{2}{|c|}{60} & 1,33 & 58,0 \\
\hline
\end{tabular}

$\mathrm{V}=$ volumoso

$\mathrm{C}=$ concentrado.

Tabela 5 - Médias e erros-padrão (EP) do número diário de mastigações, número de bolos mastigados por dia e tempo de mastigação diária

\begin{tabular}{|c|c|c|c|c|c|c|}
\hline \multirow[b]{2}{*}{ Categoria } & \multicolumn{4}{|c|}{ Frequências do fornecimento da dieta } & \multirow[b]{2}{*}{ EP } & \multirow[b]{2}{*}{ Médias } \\
\hline & $2 \mathrm{~V} / \mathrm{C}$ & $1 \mathrm{~V} / \mathrm{C}$ & $1 \mathrm{~V} / 2 \mathrm{C}$ & $1 \mathrm{~V} / 3 \mathrm{C}$ & & \\
\hline \multicolumn{7}{|c|}{ Número de bolos/dia } \\
\hline Vacas & $507 a b$ & $553 a$ & 436b & $485 a b$ & 27,38 & $495 \pm 13,69$ \\
\hline Novilhas & $540 \mathrm{a}$ & $512 \mathrm{ab}$ & $563 a$ & $541 \mathrm{a}$ & 27,38 & $539 \pm 13,69$ \\
\hline Médias & 523 & 532 & 499 & 513 & 19,36 & \\
\hline \multicolumn{7}{|c|}{ Número de mastigadas/dia } \\
\hline Vacas & 30.690 & 28.453 & 25.951 & 25.571 & $1.301,8$ & $27.666 \pm 650,9 \mathrm{~B}$ \\
\hline Novilhas & 33.826 & 29.340 & 30.947 & 31.752 & $1.301,8$ & $31.466 \pm 650,9 \mathrm{~A}$ \\
\hline Médias & $32.258 \mathrm{~A}$ & $28.896 \mathrm{~B}$ & 28.449B & $28.662 \mathrm{~B}$ & 920,53 & \\
\hline \multicolumn{7}{|c|}{ Tempo de mastigação, horas/dia } \\
\hline Vacas & $13,07 \mathrm{a}$ & $13,44 \mathrm{a}$ & $11,84 \mathrm{~b}$ & $11,82 b$ & 0,21 & $12,54 \pm 0,10$ \\
\hline Novilhas & $13,16 a$ & $12,41 b$ & $12,42 b$ & $11,94 b$ & 0,21 & $12,48 \pm 0,10$ \\
\hline Médias & 13,12 & 12,92 & 12,13 & 11,88 & 0,15 & \\
\hline
\end{tabular}

a,b Médias seguidas por letras minúsculas ou maiúsculas diferentes para mesmo parâmetro diferem $(\mathrm{P}<0,05)$ entre si.

$\mathrm{V}=$ volumoso.

$\mathrm{C}=$ concentrado 
As novilhas e os animais que receberam duas vezes 0 volumoso e o concentrado ao dia apresentaram maior número de mastigadas mericíclicas diárias. As novilhas apresentaram maior número de mastigações por bolo e maior número de bolos mastigados por dia, o que refletiu em maior número de mastigadas mericíclicas diárias.

O tempo de mastigação diária, que envolve o tempo de alimentação e ruminação, foi maior $(\mathrm{P}<0,05)$ para as vacas que receberam uma vez o volumoso e o concentrado em comparação as novilhas, valores de 13,44 e 12,41 horas, respectivamente. Estes valores encontram-se próximos ao verificados por Missio et al. (2010) para animais recebendo uma dieta com $40 \%$ de concentrado e $60 \%$ de silagem de milho. Os ruminantes podem reduzir a duração do tempo de mastigações diária pelo aumento da eficiência na redução das partículas (Deswysen et al., 1987), pela diminuição da proporção dos movimentos mandibulares em relação ao número de movimentos totais (Deswysen \& Erhlein, 1981), pela redução no intervalo entre os bolos ruminais (Gordon, 1965), pelo aumento na taxa de movimentos mandibulares (Bae et al., 1981), ou pela interação destes.

O tempo consumindo alimento não teve variação $(\mathrm{P}>0,05)$ conforme a frequência do fornecimento da dieta (Tabela 6). A taxa de ingestão de alimento, que é expressa pelo CMS em relação ao tempo consumindo alimento, foi superior $(\mathrm{P}<0,05)$ para os animais que receberam uma vez o volumoso e três vezes o concentrado, e esta não diferindo dos que receberam $1 \mathrm{~V} / 2 \mathrm{C}$ em relação ao fornecimento de 1 V/C e 2 V/C (2,89; 2,72; 2,43 e 2,48 kg de MS/hora de consumo, respectivamente). A maior taxa de ingestão de alimento é consequência do maior CMS para estes animais (Tabela 2), já que o tempo consumindo alimento não teve variação. De acordo com Thiago et al. (1992), a quantidade de alimento consumido pelo ruminante, em determinado período de tempo, depende do número de refeições nesse período e da duração e taxa de alimentação de cada refeição. Cada um desses processos é o resultado da interação do metabolismo do animal e das propriedades físicas e químicas da dieta, estimulando receptores da saciedade. Ferreira (2006) não verificou diferença $(\mathrm{P}>0,05)$ no tempo consumindo alimento, taxa de ingestão de alimento e CMS, quando avaliou dois, três ou quatro fornecimentos da dieta. Silva et al. (2005) afirmaram que a eficiência de alimentação depende da magnitude de variação do teor dos componentes fibrosos da dieta. Neste estudo, a dieta apresentava a mesma composição de nutrientes para as diferentes frequências de fornecimento. Assim, o teor dos componentes fibrosos não variou, e o que influenciou a taxa de ingestão foi o maior consumo de alimento para os animais que receberam o fornecimento do concentrado duas ou três vezes.

Bürger et al. (2000) verificaram que a taxa de ingestão aumentou de forma linear $(\mathrm{P}<0,05)$ de acordo com os níveis de concentrado na dieta (30; 45; 60; 75 e 90\% de concentrado), reflexo do peso específico e do teor de FDN do alimento, já que nas dietas com maiores proporções de concentrado o peso específico do alimento foi maior, devido à maior participação da fração concentrada, e com menor teor de FDN, o que proporcionou que o animal captasse maior quantidade de alimento em menor tempo.

O tempo de ingestão de água foi maior para os animais que receberam $1 \mathrm{~V} / 2 \mathrm{C} \mathrm{e} 1 \mathrm{~V} / 3 \mathrm{C}$ em relação ao fornecimento de $2 \mathrm{~V} / \mathrm{C}$ e $1 \mathrm{~V} / \mathrm{C}(0,27 ; 0,31 ; 0,17$ e 0,17 hora, respectivamente), provavelmente como influência do maior consumo de MS nestas frequências de fornecimento (Tabela 2). Ferreira (2006) também encontrou diferenças para o tempo de ingestão de água, onde três refeições diárias levaram os animais a ingerir água por mais tempo em relação as duas ou quarto refeições. A maior influência no consumo de água, segundo o NRC (2001), é do tipo e da concentração de MS no alimento, da temperatura ambiente e do nível de produção do animal.

As vacas permaneceram mais tempo consumindo alimento em relação às novilhas (Tabela 7), apresentando valores de 4,38 e 4,09 horas, respectivamente, influenciado pelo maior consumo de alimento das vacas (Tabela 2).

A taxa de ingestão de alimento foi maior $(\mathrm{P}<0,05)$ para as vacas em relação às novilhas, valores de 2,91 e 2,35 kg de $\mathrm{MS} / \mathrm{h}$, respectivamente, devido ao maior tamanho do bocado das mesmas. Segundo Van Soest (1994), a eficiência

Tabela 6 - Médias e erros-padrão (EP) dos tempos consumindo alimento e bebendo água e taxa de ingestão de alimento

\begin{tabular}{|c|c|c|c|c|c|c|}
\hline \multirow[b]{2}{*}{ Variável } & \multicolumn{4}{|c|}{ Frequência do fornecimento da dieta } & \multirow[b]{2}{*}{ EP } & \multirow[b]{2}{*}{ Médias } \\
\hline & $2 \mathrm{~V} / \mathrm{C}$ & $1 \mathrm{~V} / \mathrm{C}$ & $1 \mathrm{~V} / 2 \mathrm{C}$ & $1 \mathrm{~V} / 3 \mathrm{C}$ & & \\
\hline Tempo consumindo alimento, h & 4,31 & 4,38 & 4,32 & 3,92 & 0,12 & 4,23 \\
\hline Tempo bebendo água, h & $0,17 b$ & $0,17 b$ & $0,27 \mathrm{a}$ & $0,31 \mathrm{a}$ & 0,01 & 0,23 \\
\hline Taxa de ingestão, kg de MS/h & $2,48 \mathrm{bc}$ & $2,43 c$ & $2,72 \mathrm{ab}$ & $2,89 a$ & 0,09 & 2,63 \\
\hline
\end{tabular}

a,b,c Médias seguidas na linha de letras diferentes diferem $(\mathrm{P}<0,05)$ entre si.

$\mathrm{V}=$ volumoso.

$\mathrm{C}=$ concentrado 
Tabela 7 - Tempos consumindo alimento e bebendo água e taxa de ingestão do alimento

\begin{tabular}{lcccc}
\hline & \multicolumn{2}{c}{ Categoria } & & Erros-padrão \\
\cline { 2 - 3 } Variável & Vacas & Novilhas & Médias \\
\hline Tempo consumindo alimento, horas & $4,38 \mathrm{a}$ & $4,09 \mathrm{~b}$ & 0,08 & 4,23 \\
Tempo bebendo água, horas & 0,23 & 0,23 & 0,009 & 0,23 \\
Taxa de ingestão, kg de MS/hora & $2,91 \mathrm{a}$ & $2,35 \mathrm{~b}$ & 0,06 & 2,63 \\
\hline
\end{tabular}

com que o animal colhe o alimento está relacionada ao tempo destinado ao consumo de alimento e ao peso específico do alimento consumido. Bürger et al. (2000) encontraram crescimento linear para taxa de ingestão de MS, encontrando valores de 1,03; 1,52; 1,75; 2,98 e 2,86 kg MS/hora para animais alimentados com 30; 45; 60; 75 e $90 \%$ de concentrado na dieta.

O fornecimento do alimento em diferentes frequências estimulou os animais a intensificar a procura e a sua ingestão (Figura 1), mas o tempo consumindo alimento não foi alterado ao longo do dia (Tabela 6).

Neste estudo, o comportamento foi semelhante ao observado por Ferreira (2006) de que a maior frequência de animais ao comedouro foi verificada no momento de fornecimento da dieta, além de número variável de pequenas refeições entre elas. Queiroz et al. (2001) verificaram que o horário de fornecimento da ração ocasionou diferença nas características de ingestão, com o tempo despendido em ingestão maior quando o alimento foi fornecido pela manhã. Miranda et al. (1999), estudando o comportamento alimentar de novilhas, observaram que a maior porção de consumo ocorreu durante o dia, uma vez que o consumo de MS foi maior após o fornecimento de alimentação fresca e a ruminação ocorre preferencialmente à noite, quando a temperatura ambiental é mais amena, o que também foi observado neste trabalho.

A influência de fatores de manejo sobre o comportamento ingestivo de bovinos é ressaltada por Mertens (1996) e Gibson (1984), que afirmaram que as atividades ingestivas são influenciadas pela distribuição da ração, estimulando o consumo. Segundo Van Soest (1994), a atividade de pastejo

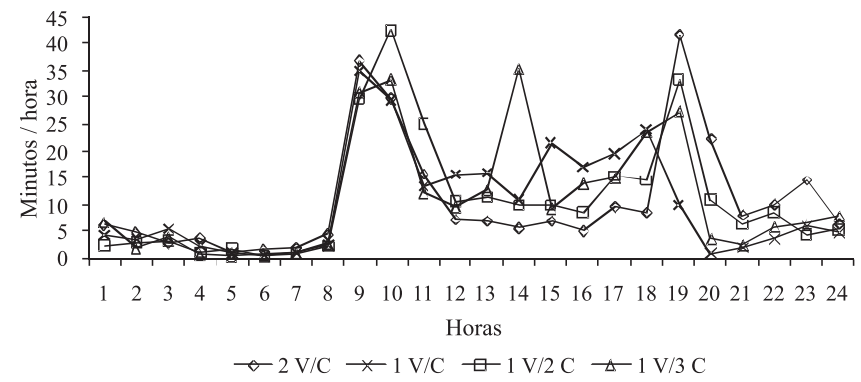

Figura 1 - Tempo de presença dos animais (minutos/hora) no cocho ao longo do dia. em bovinos é mais acentuada pela manhã e à tarde, e a ruminação ocorre à noite, embora possa ocorrer ruminação por volta do meio-dia. Souza et al. (2007), avaliando o comportamento ingestivo diurno de bovinos em pastagens, observaram que o percentual gasto em ruminação foi baixo $(8,97 \%)$, comprovando que esses animais aproveitaram a fase diurna para se alimentar (20,69\%) ruminando à noite. Já em confinamento, Fischer et al. (1998) avaliaram o fornecimento do alimento duas vezes ao dia e relataram que as refeições que seguem a distribuição do alimento são as mais importantes e duram cada uma de 1 a 3 horas. Freitas et al. (2010), avaliando a presença dos animais no comedouro, verificaram maior presença nos horários próximo ao fornecimento da alimentação ( 8 h 30 min e às $14 \mathrm{~h}$ ) e ao entardecer, demonstrando o condicionamento dos animais a rotina de manejo.

\section{Conclusões}

A frequência do fornecimento do volumoso e do concentrado a vacas e novilhas em uma, duas ou três vezes ao dia estimula esses animais a intensificar a ingestão no momento do fornecimento, mas isso não altera o tempo de ingestão do alimento ao longo do dia, o consumo de matéria seca (\% do peso corporal) e o ganho de peso e ainda promove maior mão-de-obra para o fornecimento do alimento. A dieta fornecida uma vez ao dia para as novilhas proporciona maior tempo em ócio e menor tempo de mastigação diário em relação às vacas, no entanto, não proporciona maior ganho de peso. As vacas apresentam maior tempo de consumo e taxa de ingestão em relação às novilhas, acarretando maior consumo de alimento.

\section{Referências}

ASSOCIATION OF OFFICIAL ANALYTICAL CHEMISTRY AOAC. Official methods of analysis. 16.ed. Washington, D.C.: 1995. 2000p.

BAE, D.H.; WELCH, J.; SMITH, A.M. Efficiency of mastication in relation to hay intake by cattle. Journal of Animal Science, v.52, p.1371-1375, 1981.

BURGER, P.J.; PEREIRA, J.C.; QUEIROZ, A.C. et al. Comportamento ingestivo em bezerros holandeses alimentados com dietas contendo diferentes níveis de concentrado. Revista Brasileira de Zootecnia, v.29, n.1, p.236-242, 2000. 
DESWYSEN，A.G.; DUTILLEUL， P.A.; GODFRIN， J.P. Nycterohemeral eating and ruminanting patterns in heifers fed grass or corn silage: analysis by finite fourier transform. Journal of Animal Science, v.71, n.10, p.2739-2747, 1993.

DESWYSEN, A.G.; EHRLEIN, H.J. Silage intake, rumination and pseudorumination activity in sheep studied by radiography and jaw movements recordings. British Journal Nutrition, v.46, p.327-336, 1981.

DESWYSEN, A.G.; ELLIS, W.C.; POND, K.R. Interrelationships among voluntary intake, eating and ruminating behavior and ruminal motility of heifers fed corn silage. Journal of Animal Science, v.64, p.835-841, 1987.

FERREIRA, J.J. Desempenho e comportamento ingestivo de novilhos e vacas sob frequências de alimentação em confinamento. 2006. 80f. Dissertação (Mestrado em Zootecnia) - Universidade Federal de Santa Maria, Santa Maria.

FISCHER, V.; DESWYSEN, A.G.; DESPRES, L. et al. Padrões nictemerais do comportamento ingestivo de ovinos. Revista Brasileira de Zootecnia, v.27, p.362-369, 1998.

FISCHER, V.; DESWYSEN, A.G.; DESPRES, P. et al. Comportamento ingestivo de ovinos recebendo dieta a base de feno durante um período de seis meses. Revista Brasileira de Zootecnia, v.5, p.1032-1038, 1997.

FISCHER, V.; DESWYSEN, A.G.; DUTILLEUL, P. et al. Padrões da distribuição nictemeral do comportamento ingestivo de vacas leiteiras, ao início e ao final da lactação, alimentadas com dieta à base de silagem de milho. Revista Brasileira de Zootecnia, v.31, n.5, p.2129-2138, 2002

FREITAS, L.S; SILVA, J.H.S.; SEGABINAZZI, L.R. et al. Substituição da silagem de milho por silagem de girassol na dieta de novilhos em confinamento: comportamento ingestivo. Revista Brasileira de Zootecnia, v.39, n.1, p.225-232, 2010.

GIBSON, J.P. The effects of frequency of feeding on milk production of dairy cattle: an analysis of published results. Animal Production, v.38, p.181-189, 1984.

KOMAREK, A.R. A fiber bag procedure for improved efficiency of fiber analyses. Journal of Dairy Science, v.76, p.250, 1993.

LIMA, R.M.B.; FERREIRA, M.A.; BRASIL, L.H.A. et al. Substituição de milho por palma forrageira: comportamento ingestivo de vacas mestiças em lactação. Acta Scientiarum. Animal Sciences, v.25, n.2, p.347-353, 2003.

MENDES, C.Q.; TURINO, V.F.; SUSIN, I. Comportamento ingestivo de cordeiros e digestibilidade dos nutrientes de dietas contendo alta proporção de concentrado e diferentes fontes de fibra em detergente neutro. Revista Brasileira de Zootecnia, v.39, n.3, p.594-600, 2010

MENDONÇA, S.S.; CAMPOS, S.C.; VALADARES FILHO, R.F.D. et al. Comportamento ingestivo de vacas leiteiras alimentadas com dietas à base de cana de açúcar ou silagem de milho. Revista Brasileira de Zootecnia, v.33, p.723-728, 2004.

MERTENS, D.R. Creating a system for meeting the fiber requirements of dairy cows. Journal of Dairy Science, v.80, n.8, p.1463-1469, 1997.
MERTENS, D.R. Methods in modeling feeding behavior and intake in herbivores. Annales Zootechnie, v.45, p.153-164, 1996.

MIRANDA, L.F.; QUEIROZ, A.C.; VALADARES FILHO, S.C. et al. Comportamento ingestivo de novilhas leiteiras alimentadas com dietas à base de cana-de-açúcar. Revista Brasileira de Zootecnia, v.28, p.614-620, 1999.

MISSIO, R.L.; BRONDANI, I.L.; ALVES FILHO, D.C. et al. Comportamento ingestivo de tourinhos terminados em confinamento, alimentados com diferentes níveis de concentrado na dieta. Revista Brasileira de Zootecnia, v.39, n.7, p.1571-1578, 2010.

MONZANO, R.P.; NUSSIO, L.G.; CAMPOS, F.P. et al. Comportamento ingestivo de novilhos sob suplementação em pastagens de capim-tanzânia sob diferentes intensidades de desfolhação. Revista Brasileira de Zootecnia, v.36, n.3, p.550-557, 2007.

NATIONAL REQUIREMENT COUNCIL - NRC. Nutrient requirements of beef cattle. 7.ed. Washington, D.C.: National Academy Press, 2001. 242p.

NATIONAL RESEARCH COUNCIL - NRC. Nutrient requirements of domestic animals. 7. rev. ed. Washington: National Academy Press, 1996. 242p.

QUEIROZ, A.C.; NEVES; J.S.; MIRANDA, L.F. et al. Efeito do nível de fibra e da fonte de proteína sobre o comportamento alimentar de novilhas mestiças Holandês-Zebu. Arquivo Brasileiro de Medicina Veterinária e Zootecnia, v.53, n.1, 2001.

SILVA, R.R.; SILVA, F.F.; CARVALHO, G.G.P. et al. Comportamento ingestivo de novilhas mestiças holandês $\mathrm{x}$ zebu confinadas. Archivos de Zootecnia, v.54, n.205, p.75-85, 2005.

SOARES, A.B.; MEZZALIRA, J.C.; BUENO, E.A.C. et. al. Efeito de diferentes intensidades de pastejo em pastagem nativa melhorada sobre o desempenho animal. Revista Brasileira de Zootecnia, v.35, n.1, p.75-83, 2006.

SOUZA, S.R.M.B.O.; ÍTAVO, L.C.V.; RIMOLI, J. et al. Comportamento ingestivo diurno de bovinos em confinamento e em pastagens. Archivos de Zootecnia, v.56, n.213, p.67-70, 2007.

STATISTICAL ANALYSiS SYSTEM - SAS. SAS Language Reference. Version 6. Cary, NC: SAS Institute, 2001. 1042p.

THIAGO, L.R.L.; GILL, M.; SISSONS, J.W. Studies of conserving grass herbage and frequency of feeding in cattle. British Journal Nutrition, v.67, n.3, p.339-336, 1992.

VAN SOEST, P.J. Nutritional ecology of the ruminant. 2.ed. Ithaca, NY, Cornell University Press, 1994. 476p.

WEISS, W.P.; CONRAD, H.R.; ST. PIERRE, N.R. A theoreticallybased model for predicting total digestible nutrient values of forages and concentrates. Animal Feed Science and Technology, v.39, p.95-110, 1992.

WELCH, J.G. Rumination, particle size and passage from the rumen. Journal of Animal Science, v.54, n.4, p.885-894, 1982.

ZANINE, A.M.; SANTOS, E.M.; PARENTE, H.N. et al. Hábito de pastejo de vacas lactantes Holandês x Zebu em pastagem de Brachiaria brizantha e Brachiaria decumbens. Arquivos Brasileiro de Medicina Veterinária e Zootecnia, v.59, n.1, p.175-181, 2007. 